\title{
Transverse momentum resummation for color sextet and antitriplet scalar production at the LHC
}

\author{
Ze Long Liu ${ }^{1}$, Chong Sheng $\mathrm{Li}^{1,2, a}$, Yan Wang ${ }^{1}$, Yong Chuan Zhan ${ }^{1}$, Hai Tao Li ${ }^{1}$ \\ ${ }^{1}$ School of Physics and State Key Laboratory of Nuclear Physics and Technology, Peking University, Beijing 100871, China \\ ${ }^{2}$ Center for High Energy Physics, Peking University, Beijing 100871, China
}

Received: 4 November 2013 / Accepted: 5 February 2014 / Published online: 26 February 2014

(C) The Author(s) 2014. This article is published with open access at Springerlink.com

\begin{abstract}
We study the factorization and resummation of the transverse momentum spectrum of the color sextet and antitriplet scalars produced at the LHC based on softcollinear effective theory. Compared to $Z$ boson and Higgs production, a soft function is required to account for the soft gluon emission from the final-state colored scalar. The soft function is calculated at the next-to-leading order, and the resummation is performed at the approximate next-tonext-to-leading logarithmic accuracy. The non-perturbative effects and PDF uncertainties are also discussed.
\end{abstract}

\section{Introduction}

The Large Hadron Collider (LHC) provides a great opportunity to search for new physics beyond the Standard Model (SM). For example, observation of the color sextet (antitriplet) scalars will be a direct signal of new physics beyond the SM. In fact, the color sextet (antitriplet) scalars have been included in many new physics models, such as unification theories [1-3], supersymmetry with R-parity violation [4], diquark Higgs et al. [5]. So it is preferable to be concerned with such a signal in a model independent way rather than considering some specific models. A colored scalar can be produced in quark-quark fusion with color structure obtained from $\mathbf{3} \times \mathbf{3}=\mathbf{6} \oplus \overline{\mathbf{3}}$, where $\mathbf{3}, \mathbf{6}$, and $\overline{\mathbf{3}}$ are the triplet, sextet and antitriplet representations of the $S U(3)_{C}$ color group. The interaction of the color sextet (antitriplet) scalars with two quarks can be written as

$\mathcal{L}=2 \sqrt{2}\left[\bar{K}_{i}^{a b} \phi^{i} \bar{\psi}_{a}\left(\lambda_{\mathrm{L}} P_{\mathrm{L}}+\lambda_{\mathrm{R}} P_{\mathrm{R}}\right) \psi_{b}^{\mathrm{C}}+\right.$ h.c. $]$,

where $P_{\mathrm{L}, \mathrm{R}} \equiv\left(1 \mp \gamma_{5}\right) / 2$ are the usual left- and right-hand projectors, $\lambda_{\mathrm{L} / \mathrm{R}}$ is the Yukawa like coupling, $a, b$ are the color indices, and $\bar{K}_{i}{ }^{a b}$ is for the Clebsch-Gordan coefficients [6].

\footnotetext{
a e-mail: csli@pku.edu.cn
}

$q^{\mathrm{C}}$ is the charge conjugate quark field, and the sum over quark flavors has been suppressed. The scalar $\phi^{i}$ transforms according to either sextet or antitriplet representation of $S U(3)_{\mathrm{C}}$. The production of a heavy resonance via quark-quark fusion is significantly enhanced at the LHC for larger values of the partonic Bjorken- $x$, because valence quarks have large parton density there, where the gluon density drops off rapidly.

The exotic colored states attract a lot attention in experiments $[7,8]$. The most current data reported by the CMS experiment [7] at $\sqrt{s}=8 \mathrm{TeV}$ corresponding to an integrated luminosity of $4 \mathrm{fb}^{-1}$ excludes $\mathrm{E}_{6}$ diquarks [9] with mass less than $4.28 \mathrm{TeV}$ at $95 \%$ confidence level. As shown in Refs. [5,10,11], the measurements of $D^{0}-\overline{D^{0}}$ mixing and the rate of $D \rightarrow \pi^{+} \pi^{0}\left(\pi^{+} \phi\right)$ decay can constrain the couplings of the colored scalars to two up-type quarks $\lambda_{\mathrm{R}}^{u u}, \lambda_{\mathrm{R}}^{u c} \leq 0.1$, $\left|\operatorname{Re}\left(\lambda^{c c} \lambda^{u u *}\right)\right| \sim 5.76 \times 10^{-7}$ for $m_{\phi} \sim 1 \mathrm{TeV}$. In addition, the left-handed coupling $\lambda_{\mathrm{L}}$ also gets tight constraints due to minimal flavor violation. Since we use the model independent coupling $\lambda^{2}=\lambda_{\mathrm{L}}^{2}+\lambda_{\mathrm{R}}^{2}$, the above constraints can be relaxed in the following scenario.

Resonant production of the color antitriplet scalars and vectors has been calculated at the leading order (LO), respectively, in Refs. [12-15], and pair production of the color sextet scalars has been studied at the LO in Refs. $[11,16]$. In Ref. [6],the next-to-leading order (NLO) QCD corrections to the production of color sextet and antitriplet scalars have also been calculated. The decay of color triplet and sextet has also been studied in Ref. [17] and Refs. [18, 19], respectively. Very recently, the threshold resummation for the production of a color sextet (antitriplet) has been investigated in Ref. [20]. As is well known in the case of Drell-Yan and Higgs production, the fixed-order predictions are unreliable in small $q_{\mathrm{T}}$ region, because soft and collinear gluon emissions give rise to large logarithms of scale ratio $\ln \left(q_{\mathrm{T}}^{2} / Q^{2}\right)$ at each order in perturbation theory, where $Q \gg q_{\mathrm{T}}$ is a typical hard scale of the process. For the case of DrellYan and Higgs, the method to deal with this problem is the 
so-called Collins-Soper-Sterman (CSS) formalism [21-23], in which the large logarithms can be resummed to all orders in the strong coupling $\alpha_{\mathrm{s}}$. For colored scalar production, the CSS formalism cannot be directly applied due to gluon emissions from the colored scalar in the final state. Nevertheless, Ref. [6] achieved the transverse momentum resummation for the production of a colored scalar at the leading logarithmic (LL) level by modifying the CSS formalism.

In this paper, we investigate the transverse momentum resummation in single production of the color sextet (antitriplet) scalars at the LHC with the approximate next-

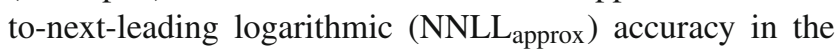
framework of the soft-collinear effective theory (SCET) [2426]. The framework is built upon the work in Refs. [27-30], which systematically resum the large logarithms to arbitrary accuracy. A novel feature of the method in the framework of SCET is the appearance of a transverse soft function, which describes color exchange among the initial-state and final-state particles.

This paper is organized as follows. In Sect. 2, we briefly show the derivation of the factorization formula for the single colored scalar production at the LHC. In Sect. 3, we calculate the hard function and the soft function at the NLO, and we show the resummation formula at the $\mathrm{NNLL}_{\text {approx }}$. We expand the resummation formula to the NLO in Sect. 4 and compare them with the exact NLO calculation at small transverse momentum. In Sect. 5, we discuss the scale and PDF uncertainties of the cross section, and we compare our numerical results with the ones in Ref. [6]. We conclude in Sect. 6.

\section{Derivation of the factorization formula}

In this section we present the derivation of the factorization for the production of a color sextet (antitriple) scalar using SCET. The transverse momentum resummation discussed has some similarity with threshold resummation [20], for example, the hard function which encodes the short-distance physics is exactly the same as the one in threshold resummation. But it is genuinely different from that, since the treatment of soft and collinear radiations are completely different from the threshold resummation.

We study the production of a colored scalar with mass $m_{\phi}$ and transverse momentum $q_{\mathrm{T}}$ in the kinematic region where $m_{\phi}^{2} \gg q_{\mathrm{T}}^{2} \gg \Lambda_{\mathrm{QCD}}^{2}$. To describe collinear and soft fields in SCET, it is convenient to define two lightlike vectors along the beam directions $n^{\mu}=(1,0,0,1)$ and $\bar{n}^{\mu}=(1,0,0,-1)$, which satisfy $n \cdot \bar{n}=2$. We can decompose any four-vector with respect to $n^{\mu}$ and $\bar{n}^{\mu}$ as

$p^{\mu}=(n \cdot p) \frac{\bar{n}^{\mu}}{2}+(\bar{n} \cdot p) \frac{n^{\mu}}{2}+p_{\perp}^{\mu}=p_{+} \frac{\bar{n}^{\mu}}{2}+p_{-} \frac{n^{\mu}}{2}+p_{\perp}^{\mu}$.
We define a small parameter $\lambda=q_{\mathrm{T}} / m_{\phi}$ and quote the components $\left(p_{+}, p_{-}, p_{\perp}\right)$ of momentum. The relevant momentum regions are

hard:

hard-collinear: $\quad p_{h c} \sim m_{\phi}\left(\lambda^{2}, 1, \lambda\right)$,

anti-hard-collinear: $p_{\overline{h c}} \sim m_{\phi}\left(1, \lambda^{2}, \lambda\right)$,

soft: $\quad p_{s} \sim m_{\phi}(\lambda, \lambda, \lambda)$.

We consider the process

$N_{1}\left(P_{1}\right)+N_{2}\left(P_{2}\right) \rightarrow \phi(q)+X\left(p_{X}\right)$,

where $N_{1}$ and $N_{2}$ are the incoming hadrons and $X$ are the inclusive hadronic final states. For later convenience, we define the following kinematic variables:

$s=\left(P_{1}+P_{2}\right)^{2}, \quad \tau=\frac{m_{\phi}^{2}+q_{\mathrm{T}}^{2}}{s}$.

Generally, the differential cross section can be written as

$$
\begin{aligned}
\mathrm{d} \sigma= & \frac{1}{2 s} \frac{\mathrm{d}^{3} \vec{q}}{(2 \pi)^{3} 2 E_{\phi}} \int d^{4} x\left\langle N_{1}\left(P_{1}\right) N_{2}\left(P_{2}\right)\left|\hat{\Phi}^{\dagger}(x)\right| \phi(q)\right\rangle \\
& \times\left\langle\phi(q)|\hat{\Phi}(0)| N_{1}\left(P_{1}\right) N_{2}\left(P_{2}\right)\right\rangle
\end{aligned}
$$

with

$\hat{\Phi}=2 \sqrt{2} K_{a b}^{i} \phi_{i}^{\dagger} \psi_{a}^{\mathrm{T}}\left(\lambda_{\mathrm{L}}^{*} P_{\mathrm{R}}+\lambda_{\mathrm{R}}^{*} P_{\mathrm{L}}\right) \psi_{b}$.

In SCET, the $n$-collinear quark $\psi_{n}$ can be written as

$\chi_{n}(x)=W_{n}^{\dagger}(x) \xi_{n}(x), \quad \xi_{n}(x)=\frac{h \hbar}{4} \psi_{n}(x)$,

where $W_{n}(x)$ is the $n$-collinear Wilson line [25], which describes the emission of arbitrary $n$-collinear gluons from an $n$-collinear quark.

At the leading power in $\lambda$, only the $n \cdot A_{s}$ component of soft gluons can interact with the $n$-collinear field. Such an interaction is eikonal and can be removed by a field redefinition [25]:

$\chi_{n}(x) \rightarrow Y_{n}(x) \chi_{n}(x), \quad \phi_{v}(x) \rightarrow Y_{v}(x) \phi_{v}(x)$,

with

$Y_{n}(x)=\mathbf{P} \exp \left(i g_{s} \int_{-\infty}^{0} \mathrm{~d} s n \cdot A_{s}^{a}(x+s n) t^{a}\right)$,

and

$Y_{v}(x)=\mathbf{P} \exp \left(-i g_{s} \int_{0}^{\infty} \mathrm{d} s v \cdot A_{s}^{a}(x+s v) t^{a}\right)$, 
where $Y_{n}(x)$ and $Y_{v}(x)$ are incoming and outgoing Wilson lines [25,31,32], respectively, and $v$ is the dimensionless vector along the directions of the momentum of the massive scalar with $v^{2}=1$. After the redefinition of the fields, the operator $\hat{\Phi}$ can be written as

$$
\hat{\Phi} \rightarrow C_{\mathrm{S}}\left(-q^{2}-i \varepsilon, \mu\right) \hat{\mathcal{O}}
$$

where

$\hat{\mathcal{O}}=2 \sqrt{2} K_{a b}^{i} Y_{v}^{\dagger} \phi_{v}^{\dagger} \chi_{\bar{n}}^{\mathrm{T}} Y_{\bar{n}} C\left(\lambda_{\mathrm{L}}^{*} P_{\mathrm{R}}+\lambda_{\mathrm{R}}^{*} P_{\mathrm{L}}\right) Y_{n} \chi_{n}$,

and $C_{\mathrm{S}}\left(-m_{\phi}^{2}-i \varepsilon, \mu\right)$ is the hard Wilson coefficient. $C$ is the charge conjugation matrix. The matrix element for the process of single colored scalar production can factorize in the form

$$
\begin{aligned}
& \left\langle N_{1}\left(P_{1}\right) N_{2}\left(P_{2}\right)\left|\hat{\mathcal{O}}^{\dagger}(x) \hat{\mathcal{O}}(0)\right| N_{1}\left(P_{1}\right) N_{2}\left(P_{2}\right)\right\rangle \\
& =\frac{2 N_{\mathrm{D}} \lambda^{2}}{N_{\mathrm{c}}^{2}}\left\langle N_{1}\left(P_{1}\right)\left|\bar{\chi}_{n}(x) \frac{\not h}{2} \chi_{n}(0)\right| N_{1}\left(P_{1}\right)\right\rangle \\
& \quad \times\left\langle N_{2}\left(P_{2}\right)\left|\bar{\chi}_{\bar{n}}(x) \frac{\hbar h}{2} \chi_{\bar{n}}(0)\right| N_{2}\left(P_{2}\right)\right\rangle \mathcal{S}(x, \mu),
\end{aligned}
$$

where

$\mathcal{S}(x, \mu)=\frac{1}{N_{\mathrm{D}}}\left\langle 0\left|\operatorname{Tr}\left[\overline{\mathbf{T}}\left(Y_{n}^{\dagger} Y_{\bar{n}}^{\dagger} Y_{v}\right)(x) \mathbf{T}\left(Y_{\bar{n}} Y_{n} Y_{v}^{\dagger}\right)(0)\right]\right| 0\right\rangle$

is the soft function. The trace is over color indices, and the time-ordering operator $\mathbf{T}$ is required to ensure the proper ordering of soft gluon fields in the soft Wilson line. $N_{\mathrm{D}}$ is the dimension of the color representation of the scalars. The initial collinear sectors in Eq. (13) can reduce to the transverse momentum dependent parton distribution functions (TMD PDFs) [27],

$$
\begin{aligned}
\mathcal{B}_{q / N_{1}}^{n}\left(z, x_{T}^{2}, \mu\right)= & \frac{1}{2 \pi} \int \mathrm{d} t \mathrm{e}^{-i z t \bar{n} \cdot p} \\
& \times\left\langle N_{1}(p)\left|\bar{\chi}_{n}\left(t \bar{n}+x_{\perp}\right) \frac{\not h}{2} \chi_{n}(0)\right| N_{1}(p)\right\rangle, \\
\mathcal{B}_{q / N_{2}}^{\bar{n}}\left(z, x_{T}^{2}, \mu\right)= & \frac{1}{2 \pi} \int \mathrm{d} t \mathrm{e}^{-i z t n \cdot p} \\
& \times\left\langle N_{2}(p)\left|\bar{\chi}_{\bar{n}}\left(t n+x_{\perp}\right) \frac{\not h}{2} \chi_{\bar{n}}(0)\right| N_{2}(p)\right\rangle,
\end{aligned}
$$

where $x_{\mathrm{T}}^{2} \equiv-x_{\perp}^{2}>0$. Note that $\mathcal{B}_{q / N_{1}}^{n}$ and $\mathcal{B}_{q / N_{2}}^{\bar{n}}$ in Eq. (15) are TMD PDFs for the quark. Now the matrix element for the process of a colored scalar production is factorized into two collinear sectors and a soft sector, which do not interact with each other. Thus, the differential cross section can be written as

$$
\begin{aligned}
\frac{\mathrm{d}^{2} \sigma}{\mathrm{d} q_{\mathrm{T}}^{2} \mathrm{~d} y}= & \frac{2 \pi N_{\mathrm{D}} \lambda^{2}\left(\mu^{2}\right)}{N_{\mathrm{c}}^{2} s} \mathcal{H}\left(m_{\phi}^{2}, \mu^{2}\right) \frac{1}{4 \pi} \int \mathrm{d}^{2} x_{\perp} \mathrm{e}^{-i q_{\perp} \cdot x_{\perp}} \\
\times & {\left[\mathcal{B}_{q / N_{1}}^{n}\left(\xi_{1}, x_{\mathrm{T}}^{2}, \mu\right) \mathcal{B}_{q^{\prime} / N_{2}}^{\bar{n}}\left(\xi_{2}, x_{\mathrm{T}}^{2}, \mu\right) \mathcal{S}\left(x_{\mathrm{T}}^{2}, \mu\right)\right.} \\
& \left.+\left(q \leftrightarrow q^{\prime}\right)\right]+\mathcal{O}\left(\frac{q_{T}^{2}}{M^{2}}\right)
\end{aligned}
$$

where $y$ is the rapidity of the colored scalar, $\xi_{1,2}=\sqrt{\tau} \mathrm{e}^{ \pm y}$, and $\mathcal{H}\left(m_{\phi}^{2}, \mu^{2}\right)$ is the hard function defined as $\mathcal{H}\left(m_{\phi}^{2}, \mu^{2}\right)=$ $\left|C_{\mathrm{S}}\left(-m_{\phi}^{2}-i \varepsilon, \mu^{2}\right)\right|^{2}$. The collinear anomalous terms can be factored out [27], and the product of the two TMD PDFs can be refactorized,

$$
\begin{aligned}
& \mathcal{B}_{q / N_{1}}^{n}\left(z_{1}, x_{\perp}, \mu\right) \mathcal{B}_{q^{\prime} / N_{2}}^{\bar{n}}\left(z_{2}, x_{\perp}, \mu\right)=\left(\frac{x_{\mathrm{T}}^{2} m_{\phi}^{2}}{4 \mathrm{e}^{-2 \gamma_{E}}}\right)^{-F_{q q^{\prime}}\left(x_{\mathrm{T}}^{2}, \mu\right)} \\
& \quad \times B_{q / N_{1}}^{n}\left(z_{1}, x_{\perp}, \mu\right) B_{q^{\prime} / N_{2}}^{\bar{n}}\left(z_{2}, x_{\perp}, \mu\right),
\end{aligned}
$$

where $F_{q q^{\prime}}$ is the same as the $F_{q \bar{q}}$ in Ref. [27]. The $B_{q / N}$ functions are intrinsically non-perturbative objects. For $x_{\mathrm{T}} \ll$ $1 / \Lambda_{\mathrm{QCD}}$, it can be matched onto the normal PDFs [27] via

$B_{q / N}\left(z, x_{\mathrm{T}}^{2}, \mu\right)=\sum_{i} \int \frac{\mathrm{d} \xi}{\xi} I_{q \leftarrow i}\left(\xi, L_{\perp}, \mu\right) f_{i / N}(z / \xi, \mu)$

with perturbatively calculable matching coefficient functions $I_{i \leftarrow j}$. Now the differential cross section can be further in a useful form

$$
\begin{aligned}
\frac{\mathrm{d}^{2} \sigma}{\mathrm{d} q_{\mathrm{T}}^{2} \mathrm{~d} y}= & \frac{2 \pi N_{\mathrm{D}} \lambda^{2}(\mu)}{N_{\mathrm{c}}^{2} s} \mathcal{H}\left(m_{\phi}^{2}, \mu^{2}\right) \sum_{i, j=q, q^{\prime}, g} \int_{\xi_{1}}^{1} \frac{\mathrm{d} z_{1}}{z_{1}} \int_{\xi_{2}}^{1} \frac{\mathrm{d} z_{2}}{z_{2}} \\
& \times C_{q q^{\prime} \leftarrow i j}\left(z_{1}, z_{2}, q_{\mathrm{T}}^{2}, m_{\phi}^{2}, \mu\right) f_{i / N_{1}}\left(\xi_{1} / z_{1}, \mu\right) \\
& \times f_{j / N_{2}}\left(\xi_{2} / z_{2}, \mu\right)
\end{aligned}
$$

with

$$
\begin{aligned}
& C_{q q^{\prime}} \leftarrow i j\left(z_{1}, z_{2}, q_{\mathrm{T}}^{2}, m_{\phi}^{2}, \mu\right) \\
&= \frac{1}{4 \pi} \int \mathrm{d}^{2} x_{\perp} \mathrm{e}^{-i q_{\perp} \cdot x_{\perp}}\left(\frac{x_{\mathrm{T}}^{2} m_{\phi}^{2}}{b_{0}^{2}}\right)^{-F_{q q^{\prime}}\left(L_{\perp}, a_{s}\right)} \\
& \quad \times I_{q \leftarrow i}^{n}\left(z_{1}, L_{\perp}, a_{S}\right) I_{q^{\prime} \leftarrow j}^{\bar{n}}\left(z_{2}, L_{\perp}, a_{S}\right) \mathcal{S}\left(L_{\perp}, a_{S}\right),
\end{aligned}
$$

where $a_{s}, L_{\perp}, b_{0}$ are defined as

$a_{s}=\frac{\alpha_{s}(\mu)}{4 \pi}, \quad L_{\perp}=\ln \frac{x_{T}^{2} \mu^{2}}{b_{0}^{2}}, \quad b_{0}=2 \mathrm{e}^{-\gamma_{E}}$. 


\section{Resummation}

\subsection{Running of the new physics coupling}

The new physics coupling $\lambda$ satisfies the renormalization group (RG) equation

$\frac{\mathrm{d} \ln \lambda}{\mathrm{d} \ln \mu}=\gamma^{\lambda}\left(\alpha_{s}\right)$,

where the one-loop level $\gamma^{\lambda}$ is given by

$\gamma_{0}^{\lambda}=-6 C_{\mathrm{F}}$.

By solving Eq. (22), we get $\lambda$ running from the scale $\mu_{\lambda}$ to the factorization scale $\mu$,

$\lambda\left(\mu^{2}\right)=\mathrm{e}^{-a_{\gamma^{\lambda}}\left(\mu_{\lambda}^{2}, \mu^{2}\right)} \lambda_{0}$,

where $\lambda_{0}$ denotes the new physics coupling at the scale $\mu_{\lambda}$. In this paper, we choose $\mu_{\lambda}=m_{\phi} \cdot a_{\gamma^{\lambda}}\left(v^{2}, \mu^{2}\right)$ is defined as $a_{\gamma^{\lambda}}\left(v^{2}, \mu^{2}\right)=-\int_{\alpha_{s}\left(v^{2}\right)}^{\alpha_{s}\left(\mu^{2}\right)} \mathrm{d} \alpha \frac{\gamma^{\lambda}(\alpha)}{\beta(\alpha)}$.

Now, the anomalous dimension of the new physics coupling is only available at the NLO, which means that the resummation for $\lambda$ is at the next-to-leading logarithmic (NLL) order.

\subsection{Hard function}

In SCET, $C_{\mathrm{S}}\left(-m_{\phi}^{2}, \mu^{2}\right)$ (here and below the negative arguments are understood with the $-i \varepsilon$ prescription) can be obtained to order $\mathcal{O}\left(\alpha_{S}\right)$ from one-loop virtual correction calculation, whose infrared divergences are subtracted in the $\overline{\mathrm{MS}}$ scheme [20]

$$
\begin{aligned}
C_{\mathrm{S}}\left(-m_{\phi}^{2}, \mu^{2}\right)=1 & +\frac{\alpha_{s}(\mu)}{4 \pi}\left[C_{\mathrm{F}}\left(-L^{2}+\frac{\pi^{2}}{6}-2\right)\right. \\
& \left.+C_{\mathrm{D}}\left(L-\frac{2}{3} \pi^{2}-1\right)\right],
\end{aligned}
$$

with

$L=\ln \frac{-m_{\phi}^{2}}{\mu^{2}}$.

$C_{\mathrm{S}}\left(-m_{\phi}^{2}, \mu^{2}\right)$ satisfies the RG equation [33]

$$
\begin{aligned}
& \frac{\mathrm{d}}{\mathrm{d} \ln \mu} C_{\mathrm{S}}\left(-m_{\phi}^{2}, \mu^{2}\right) \\
& =\left[\Gamma_{\text {cusp }}^{\mathrm{F}}\left(\alpha_{s}\right) \ln \frac{-m_{\phi}^{2}}{\mu^{2}}+2 \gamma^{q}\left(\alpha_{s}\right)+\gamma^{D}\left(\alpha_{s}\right)-\gamma^{\lambda}\left(\alpha_{s}\right)\right] \\
& \quad \times C_{\mathrm{S}}\left(-m_{\phi}^{2}, \mu^{2}\right) .
\end{aligned}
$$

$\Gamma_{\text {cusp }}^{F}\left(\alpha_{s}\right)$ is the cusp anomalous dimension in the fundamental representation. $\gamma^{q}$ (equal to $\gamma^{V} / 2$ in Ref. [34]) is the anomalous dimension of massless quark, and $\gamma^{D}$ is the one of colored scalar, which is given by [33]

$\gamma_{0}^{\mathrm{D}}=-2 C_{\mathrm{D}}$

$\gamma_{1}^{\mathrm{D}}=C_{\mathrm{D}} C_{\mathrm{A}}\left(\frac{2 \pi^{2}}{3}-\frac{98}{9}-4 \zeta_{3}\right)+\frac{40}{9} C_{\mathrm{D}} T_{\mathrm{F}} n_{f}$.

From now on, the coupling $\alpha_{s}$ without an explicit argument will always refer to $\alpha_{s}(\mu)$.

The solution of Eq. (28) is

$$
\begin{aligned}
& C_{\mathrm{S}}\left(-m_{\phi}^{2}, \mu^{2}\right) \\
& =\exp \left[2 S\left(\mu_{h}^{2}, \mu^{2}\right)-a_{\Gamma}\left(\mu_{h}^{2}, \mu^{2}\right) \ln \frac{-m_{\phi}^{2}}{\mu_{h}^{2}}-a_{\gamma^{\mathrm{H}}}\left(\mu_{h}^{2}, \mu^{2}\right)\right. \\
& \left.\quad+a_{\gamma^{\lambda}}\left(\mu_{h}^{2}, \mu^{2}\right)\right] C_{\mathrm{S}}\left(-m_{\phi}^{2}, \mu_{h}^{2}\right),
\end{aligned}
$$

where $\gamma^{\mathrm{H}}=2 \gamma^{q}+\gamma^{\mathrm{D}}, \mu_{h}$ is the hard matching scale and $S\left(v^{2}, \mu^{2}\right)$ is defined as

$S\left(v^{2}, \mu^{2}\right)=-\int_{\alpha_{S}\left(v^{2}\right)}^{\alpha_{s}\left(\mu^{2}\right)} \mathrm{d} \alpha \frac{\Gamma_{\mathrm{cusp}}^{F}(\alpha)}{\beta(\alpha)} \int_{\alpha_{s}\left(v^{2}\right)}^{\alpha} \frac{\mathrm{d} \alpha^{\prime}}{\beta\left(\alpha^{\prime}\right)}$.

$a_{\gamma^{\mathrm{H}}}$ and $a_{\gamma^{\lambda}}$ have a similar expression as (25). Up to NNLL, three-loop $\Gamma_{\text {cusp }}$ and two-loop normal anomalous dimension are required, and the explicit expressions of them are collected in the appendix of Ref. [34].

\subsection{Soft function}

Because the colored scalar in the final state can interact with gluon, the soft function is not trivial any more, which is different from the case of Drell-Yan. At NLO, the diagrams of calculating in eikonal approximation are shown in Fig. 1. In Ref. [27], it has been shown that the contribution from Fig. 1a vanishes because the relevant integral is scaleless. The contributions from Fig. 1b, c are given by

$$
\begin{aligned}
& \mathcal{S}_{b}^{(1)}\left(x_{\mathrm{T}}^{2}, \mu\right)=\frac{2 g_{s}^{2} \mu^{2 \epsilon}}{(2 \pi)^{3-2 \epsilon}} \frac{1}{2} C_{\mathrm{D}} \\
& \quad \times \int \mathrm{d}^{4-2 \epsilon} k \delta\left(k^{2}\right) \theta\left(k^{0}\right) \mathrm{e}^{-i k_{\perp} \cdot x_{\perp}}\left(\frac{\nu_{2}}{\bar{n} \cdot k}\right)^{\beta} \frac{n \cdot v}{(n \cdot k)(v \cdot k)}, \\
& \mathcal{S}_{c}^{(1)}\left(x_{\mathrm{T}}^{2}, \mu\right)=\frac{2 g_{s}^{2} \mu^{2 \epsilon}}{(2 \pi)^{3-2 \epsilon}} \frac{1}{2} C_{\mathrm{D}} \\
& \quad \times \int \mathrm{d}^{4-2 \epsilon} k \delta\left(k^{2}\right) \theta\left(k^{0}\right) \mathrm{e}^{-i k_{\perp} \cdot x_{\perp}}\left(\frac{\nu_{2}}{\bar{n} \cdot k}\right)^{\beta} \frac{\bar{n} \cdot v}{(\bar{n} \cdot k)(v \cdot k)},
\end{aligned}
$$

where the analytic regularization method [27] is used. After calculating the integrals in Eq. (32), we find that $\mathcal{S}_{b}^{(1)}+\mathcal{S}_{c}^{(1)}$ 
Fig. 1 One-loop diagrams contributing to the soft function $\mathcal{S}\left(x_{\mathrm{T}}, \mu\right)$. The contributions from diagrams $\mathbf{a}-\mathbf{d}$ are denoted $\mathcal{S}_{a}^{(1)}, \mathcal{S}_{b}^{(1)}, \mathcal{S}_{c}^{(1)}$, and $\mathcal{S}_{d}^{(1)}$. The vertical lines indicate cut propagators

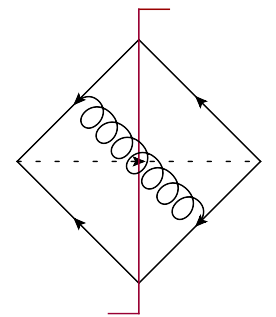

(a)

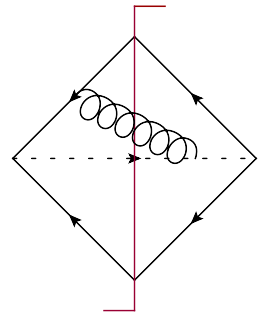

(b)

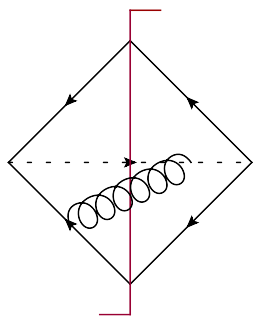

(c)

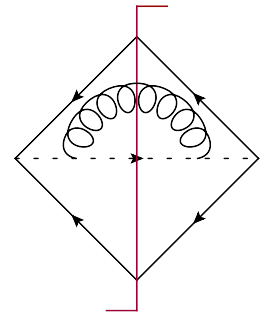

(d) is equal to zero. Therefore the soft function only depends on the contribution from Fig. 1d,

$$
\begin{aligned}
\mathcal{S}^{(1)}\left(x_{\mathrm{T}}^{2}, \mu\right)= & \mathcal{S}_{d}^{(1)}\left(x_{\mathrm{T}}^{2}, \mu\right) \\
= & -\frac{g_{s}^{2} \mu^{2 \epsilon}}{(2 \pi)^{3-2 \epsilon}} C_{\mathrm{D}} \int \mathrm{d}^{4-2 \epsilon} k \delta\left(k^{2}\right) \theta\left(k^{0}\right) \\
& \times \mathrm{e}^{-i k_{\perp} \cdot x_{\perp}}\left(\frac{\nu_{2}}{\bar{n} \cdot k}\right)^{\beta} \frac{v^{2}}{(v \cdot k)^{2}},
\end{aligned}
$$

and in the $\overline{\mathrm{MS}}$ scheme, the NLO soft function is

$\mathcal{S}\left(x_{\mathrm{T}}^{2}, \mu\right)=1+\frac{\alpha_{s} C_{\mathrm{D}}}{2 \pi} \ln \frac{x_{\mathrm{T}}^{2} \mu^{2}}{4 \mathrm{e}^{-2 \gamma_{\mathrm{E}}}}+\mathcal{O}\left(\alpha_{s}^{2}\right)$.

The RG equation of the soft function is

$\frac{\mathrm{d} \ln \mathcal{S}\left(x_{\mathrm{T}}^{2}, \mu\right)}{\mathrm{d} \ln \mu}=2 \gamma^{S_{q q}}\left(\alpha_{s}\right)$,

where $\gamma^{S_{q q}}$ is the anomalous dimension of the soft function, which can be obtained at one-loop level from Eq. (34).

In Ref. [28], the double logarithmic terms of the function $I_{q \leftarrow q}$ can be resummed by defining a new function $\bar{I}_{q \leftarrow q}$. The logarithmic term in the soft function can be resummed in the same way,

$\mathcal{S}\left(L_{\perp}, a_{S}\right) \equiv \mathrm{e}^{h_{S}\left(L_{\perp}, a_{s}\right)} \overline{\mathcal{S}}\left(L_{\perp}, a_{S}\right)$.

From the RG equation of the soft function (35), we obtain

$\frac{\mathrm{d}}{\mathrm{d} \ln \mu} h_{S}\left(L_{\perp}, a_{S}(\mu)\right)=2 \gamma^{S_{q q}}\left(a_{S}(\mu)\right)$,

$\frac{\mathrm{d}}{\mathrm{d} \ln \mu} \overline{\mathcal{S}}\left(L_{\perp}, a_{s}(\mu)\right)=0$

Matching to the NLO result of $\mathcal{S}\left(L_{\perp}, a_{S}(\mu)\right)$, we get $\overline{\mathcal{S}}\left(L_{\perp}, a_{s}(\mu)\right)=\mathcal{S}\left(0, a_{s}(\mu)\right)$ by choosing the boundary condition as $h_{S}\left(0, a_{s}(\mu)\right) \equiv 0$. Generalizing the NLO result to high orders, $h_{S}\left(L_{\perp}, a_{s}(\mu)\right)$ can be expanded,

$h_{S}\left(L_{\perp}, a_{S}(\mu)\right)=\sum_{n=1}^{\infty} h_{S}^{(n)}\left(L_{\perp}\right) a_{S}(\mu)^{n}$. $\frac{\mathrm{d} F_{q q^{\prime}}\left(x_{T}^{2}, \mu\right)}{\mathrm{d} \ln \mu}=2 \Gamma_{\text {cusp }}^{\mathrm{F}}\left(\alpha_{s}\right)$.

Using the RG equation of the soft function, we get the first two expansion coefficients of $h_{S}$,

$h_{S}^{(1)}\left(L_{\perp}\right)=\gamma_{0}^{S_{q q}} L_{\perp}$,

$h_{S}^{(2)}\left(L_{\perp}\right)=\frac{1}{2} \gamma_{0}^{S_{q q}} \beta_{0} L_{\perp}^{2}+\gamma_{1}^{S_{q q}} L_{\perp}$.

\subsection{Scale independence}

In the factorization formalism, we have introduced the hard and soft function. It is important to check the scale independence of the final results at one-loop level. As shown in Ref. [27], the RG equation for the PDFs is

$\frac{\mathrm{d}}{\mathrm{d} \ln \mu} f_{i / N}(z, \mu)=\sum_{j} \int_{z}^{1} \frac{\mathrm{d} u}{u} \mathcal{P}_{i \leftarrow j}(z / u, \mu) f_{j / N}(u, \mu)$,

the evolution equations for the kernel function $I_{q \leftarrow i}\left(z, x_{\mathrm{T}}^{2}, \mu\right)$ are

$$
\begin{aligned}
& \frac{\mathrm{d}}{\mathrm{d} \ln \mu} I_{q \leftarrow i}\left(z, x_{\mathrm{T}}^{2}, \mu\right) \\
& =\left[\Gamma_{\text {cusp }}^{\mathrm{F}}\left(\alpha_{s}\right) \ln \frac{x_{\mathrm{T}}^{2} \mu^{2}}{4 \mathrm{e}^{-2 \gamma_{E}}}-2 \gamma^{q}\left(\alpha_{s}\right)\right] I_{q \leftarrow i}\left(z, x_{\mathrm{T}}^{2}, \mu\right) \\
& \quad-\sum_{j} \int_{z}^{1} \frac{\mathrm{d} u}{u} I_{q \leftarrow j}\left(u, x_{\mathrm{T}}^{2}, \mu\right) \mathcal{P}_{j \leftarrow i}(z / u, \mu),
\end{aligned}
$$

and the RG equation for $F_{q q^{\prime}}\left(x_{T}^{2}, \mu\right)$ is

The RG invariance requires

$$
\begin{aligned}
& \frac{\mathrm{d}}{\mathrm{d} \ln \mu}\left[\lambda^{2}(\mu) \mathcal{H}\left(\mu^{2}\right)\left(\frac{x_{\mathrm{T}}^{2} m_{\phi}^{2}}{b_{0}^{2}}\right)^{-F_{q q^{\prime}}\left(L_{\perp}\right)}\right. \\
& \left.\quad \times I_{q \leftarrow i}^{n}\left(L_{\perp}\right) I_{q^{\prime} \leftarrow j}^{\bar{n}}\left(L_{\perp}\right) f_{i / N_{1}}(\mu) f_{j / N_{2}}(\mu) \mathcal{S}\left(L_{\perp}\right)\right]=0,
\end{aligned}
$$


which implies

$\gamma^{H}-2 \gamma^{q}+\gamma^{S_{q q}}=0$,

where the RG equations of the new physics coupling $\lambda$ (22), and the hard (28) and soft function (35) have been used. We confirm that Eq. (44) is satisfied at one-loop level, through calculating the anomalous dimension $\gamma^{H}$ and $\gamma^{S_{q q}}$ up to $\mathcal{O}\left(\alpha_{s}\right)$.

Using Eq. (44), the two-loop anomalous dimension of the soft function can be derived from

$\gamma^{S_{q q}}=-\gamma^{D}$

where $\gamma^{D}$ is available up to $\mathcal{O}\left(\alpha_{s}^{2}\right)(29)$.

\subsection{Final RG improved differential cross section}

Now we obtain the differential cross section of the transverse momentum resummation,

$$
\begin{aligned}
\frac{\mathrm{d}^{2} \sigma}{\mathrm{d} q_{\mathrm{T}}^{2} \mathrm{~d} y}= & \frac{2 \pi N_{\mathrm{D}} \lambda_{0}^{2}}{N_{\mathrm{c}}^{2} s} \mathcal{H}\left(m_{\phi}^{2}, \mu_{h}^{2}\right) U\left(m_{\phi}^{2}, \mu_{h}^{2}, \mu^{2}\right) \\
& \times \sum_{i, j=q, q^{\prime}, g_{\xi_{1}}} \int_{\xi_{1}}^{1} \frac{\mathrm{d} z_{1}}{z_{1}} \int_{\xi_{2}}^{1} \frac{\mathrm{d} z_{2}}{z_{2}} C_{q q^{\prime} \leftarrow i j}\left(z_{1}, z_{2}, q_{\mathrm{T}}^{2}, m_{\phi}^{2}, \mu\right) \\
& \times f_{i / N_{1}}\left(\xi_{1} / z_{1}, \mu\right) f_{j / N_{2}}\left(\xi_{2} / z_{2}, \mu\right),
\end{aligned}
$$

with

$$
\begin{aligned}
U\left(m_{\phi}^{2}, \mu_{h}^{2}, \mu^{2}\right)= & \exp \left[4 S\left(\mu_{h}^{2}, \mu^{2}\right)-2 a_{\Gamma}\left(\mu_{h}^{2}, \mu^{2}\right) \ln \frac{m_{\phi}^{2}}{\mu_{h}^{2}}\right. \\
& \left.-2 a_{\gamma^{H}}\left(\mu_{h}^{2}, \mu^{2}\right)-2 a_{\gamma^{\lambda}}\left(\mu_{\lambda}^{2}, \mu_{h}^{2}\right)\right],
\end{aligned}
$$

and

$$
\begin{aligned}
& C_{q q^{\prime} \leftarrow i j}\left(z_{1}, z_{2}, q_{\mathrm{T}}^{2}, m_{\phi}^{2}, \mu\right) \\
& =\frac{1}{2} \int_{0}^{\infty} \mathrm{d} x_{\mathrm{T}} x_{\mathrm{T}} J_{0}\left(x_{\mathrm{T}} q_{\mathrm{T}}\right) \\
& \quad \times \exp \left[g_{\mathrm{F}}\left(m_{\phi}^{2}, \mu, L_{\perp}, a_{s}\right)+h_{S}\left(L_{\perp}, a_{s}\right)\right] \\
& \quad \times \bar{I}_{q \leftarrow i}^{n}\left(z_{1}, L_{\perp}, a_{s}\right) \bar{I}_{q \leftarrow j}^{\bar{n}}\left(z_{2}, L_{\perp}, a_{s}\right) \overline{\mathcal{S}}\left(L_{\perp}, a_{s}\right),
\end{aligned}
$$

where $J_{0}$ is the zeroth order Bessel function. The expressions of $\bar{I}_{q \leftarrow i}$ and $g_{F}$ have been shown in Ref. [28].

Table 1 shows the counting scheme for resummation [34]. Up to NNLL, all the required anomalous dimensions are available, except for the two-loop $\gamma^{\lambda}$. It can only be obtained from the calculation of the two-loop $\beta$ function of the new physics coupling $\lambda$, the result of which is not available and needs to be studied in the future. Thus, we just use the oneloop $\gamma^{\lambda}$ in this paper. Actually, the contribution from $\gamma^{\lambda}$ to the evolution function $U\left(m_{\phi}^{2}, \mu_{h}^{2}, \mu^{2}\right)$ vanishes when $\mu_{h}^{2} \sim m_{\phi}^{2}$, so $\gamma^{\lambda}$ only affects the running of $\lambda\left(\mu_{\lambda}^{2}\right)$, and our resummation is called NNLL $L_{\text {approx }}$.

To give a precise prediction, we resum the singular terms to all orders and include the non-singular terms up to the NLO, which can be written as

$$
\begin{aligned}
& \frac{\mathrm{d} \sigma^{\mathrm{NNLL}} \mathrm{approx}+\mathrm{NLO}}{\mathrm{d} q_{\mathrm{T}}}=\frac{\mathrm{d} \sigma^{\mathrm{NNLL}} \text { approx }}{\mathrm{d} q_{\mathrm{T}}} \\
& +\left(\frac{\mathrm{d} \sigma^{\mathrm{NLO}}}{\mathrm{d} q_{\mathrm{T}}}-\left.\frac{\mathrm{d} \sigma^{\mathrm{NNLL}}{ }_{\text {approx }}}{\mathrm{d} q_{\mathrm{T}}}\right|_{\text {expandedtoNLO }}\right) .
\end{aligned}
$$

\section{The $q_{\mathrm{T}}$ spectrum of colored scalar at fixed order}

To verify the correctness of our factorization formula and soft functions, we expand our $q_{\mathrm{T}}$ spectrum to the NLO and compare with the exact NLO results. By expanding $C_{q q^{\prime} \leftarrow i j}$ to order $\mathcal{O}\left(\alpha_{s}\right)$ in the limit $q_{\mathrm{T}} \rightarrow 0$, the differential cross section can be written as

$$
\begin{aligned}
& \frac{\mathrm{d}^{2} \sigma}{\mathrm{d} q_{\mathrm{T}}^{2} \mathrm{~d} y}=\frac{2 \pi N_{\mathrm{D}} \lambda^{2}}{N_{\mathrm{c}}^{2} s}\left\{f_{q / N_{1}}\left(\xi_{1}\right) f_{q^{\prime} / N_{2}}\left(\xi_{2}\right)\right. \\
& \times\left(A\left[\frac{1}{q_{\mathrm{T}}^{2}} \ln \frac{m_{\phi}^{2}}{q_{\mathrm{T}}^{2}}\right]_{\star}^{\left[q_{\mathrm{T}}^{2}, \mu^{2}\right]}+B\left[\frac{1}{q_{\mathrm{T}}^{2}}\right]_{\star}^{\left[q_{\mathrm{T}}^{2}, \mu^{2}\right]}+C \delta\left(q_{\mathrm{T}}^{2}\right)\right) \\
& +\left[\sum_{a}\left(\frac{\alpha_{s}}{4 \pi}\right)\left(\frac{1}{2} P_{q \leftarrow a}\left[\frac{1}{q_{\mathrm{T}}^{2}}\right]_{\star}^{\left[q_{\mathrm{T}}^{2}, \mu^{2}\right]}+R_{q \leftarrow a} \delta\left(q_{\mathrm{T}}^{2}\right)\right)\right. \\
& \left.+f_{q / N_{1}}\left(\xi_{1}\right)\left[\sum_{a / N_{1}}\right]\left(\frac{\alpha_{s}}{4 \pi}\right)\left(\frac{1}{2} P_{q^{\prime} \leftarrow a}\left[\frac{1}{q_{\mathrm{T}}^{2}}\right]_{\star}\right]_{\star}\right]_{\mathrm{T}}, \mu_{2}\left(\xi_{2}\right) \\
& \left.\left.\left.+R_{q^{\prime} \leftarrow a} \delta\left(q_{\mathrm{T}}^{2}\right)\right) \otimes f_{a / N_{2}}\right]\left(\xi_{2}\right)+\left(q \leftrightarrow q^{\prime}\right)\right\},
\end{aligned}
$$

with

$$
\begin{gathered}
A=\frac{\alpha_{s}}{4 \pi} 4 C_{\mathrm{F}}, \quad B=-\frac{\alpha_{s}}{4 \pi}\left(6 C_{\mathrm{F}}+2 C_{\mathrm{D}}\right), \\
C=\mathcal{H}^{(1)} \mathcal{S}^{(0)}+\left.\mathcal{H}^{(0)} \mathcal{S}^{(1)}\right|_{L_{\perp} \rightarrow 0},
\end{gathered}
$$

where $P_{q \leftarrow a}$ are the NLO DGLAP splitting functions:

$$
\begin{aligned}
& P_{q \leftarrow q}(z)=4 C_{\mathrm{F}}\left(\frac{1+z^{2}}{1-z}\right)_{+}, \\
& P_{q \leftarrow g}(z)=4 T_{\mathrm{F}}\left[z^{2}+(1-z)^{2}\right],
\end{aligned}
$$


Table 1 Schemes for resummation with different level of accuracy

\begin{tabular}{lllll}
\hline Log. approx. & Accuracy $\sim \alpha_{s}^{n} L^{k}$ & $\Gamma_{\text {cusp }}$ & $\gamma^{D}, \gamma^{q}, \gamma^{\lambda}$ & $C_{\mathrm{S}}, \mathcal{S}$ \\
\hline LL & $k=2 n$ & 1-loop & Tree-level & Tree-level \\
NLL & $2 n-1 \leq k \leq 2 n$ & 2-loop & 1-loop & Tree-level \\
NNLL & $2 n-3 \leq k \leq 2 n$ & 3-loop & 2-loop & 1-loop \\
\hline
\end{tabular}

and the remainder functions $R_{q \leftarrow a}$ are

$R_{q \leftarrow q}(z)=C_{\mathrm{F}}\left(2(1-z)-\frac{\pi^{2}}{6} \delta(1-z)\right)$,

$R_{q \leftarrow g}(z)=4 T_{\mathrm{F}} z(1-z)$.

The star distribution in Eq. (49) is defined as [35,36]

$[f(x)]_{\star}^{[x, a]}=f(x)$ for $x>0$,

$\int_{0}^{a} \mathrm{~d} x[f(x)]_{\star}^{[x, a]} g(x)=\int_{0}^{a} \mathrm{~d} x f(x)[g(x)-g(0)]$.

Now we try to reproduce the NLO total cross section for colored scalar production. Using the phase space slicing method, the NLO total cross section can be divided into two parts: small $q_{\mathrm{T}}$ region denoted by $\sigma_{\mathrm{I}}$, which can be obtained by integrating the differential cross section in Eq. (49) in the approximation of neglecting $\mathcal{O}\left(q_{\mathrm{T}}^{2} / m_{\phi}^{2}\right)$ terms, and the large $q_{\mathrm{T}}$ part denoted by $\sigma_{\mathrm{II}}$, which is infrared safe and can be numerically computed directly. Thus the total cross section is given by

$\sigma_{\mathrm{NLO}}=\int_{0}^{q_{\mathrm{T}, \mathrm{cut}}^{2}} \mathrm{~d} q_{\mathrm{T}}^{2} \frac{\mathrm{d} \sigma_{\mathrm{NLO}}}{\mathrm{d} q_{\mathrm{T}}^{2}}+\int_{q_{\mathrm{T}, \text { cut }}}^{\infty} \mathrm{d} q_{\mathrm{T}}^{2} \frac{\mathrm{d} \sigma_{\mathrm{NLO}}}{\mathrm{d} q_{\mathrm{T}}^{2}}=\sigma_{\mathrm{I}}+\sigma_{\mathrm{II}}$.

As shown Fig. 2, our numerical results indicate the correctness of the hard and soft function. It can be seen that the dependence on $q_{\mathrm{T}}$, cut is canceled after summing $\sigma_{\mathrm{I}}$ and $\sigma_{\mathrm{II}}$, and the NLO total cross section is agreement with the one in Ref. [6].

\section{Numerical discussion}

In this section, we give the numerical results for the transverse momentum resummation effects in the single production of the color sextet (antitriplet) scalars at the LHC. Throughout the numerical calculation, we use MSTW 2008NLO [38] PDF sets for NLO and NLL, and we use MSTW2008NNLO PDF sets for NNLL approx $_{\text {In }}$ addition, we factored out the new physics coupling $\lambda_{0}^{2}$ for a model independent presentation and choose the initial-state quarks $u u$ for sextet and $u d$ for antitriplet, respectively. We choose the factorization scale [28]

$\mu=q_{\mathrm{T}}+q_{*}$,

where

$q_{*}=m_{\phi} \exp \left(-\frac{2 \pi}{\Gamma_{0}^{\mathrm{F}} \alpha_{S}\left(q_{*}\right)}\right)$.

From the Eq. (56), we obtain $q_{*}=2.9 \mathrm{GeV}$ for $m_{\phi}=$ $500 \mathrm{GeV}$ and $q_{*}=3.8 \mathrm{GeV}$ for $m_{\phi}=1 \mathrm{TeV}$, both of which are short-distance scales in the perturbative domain.

Besides, we choose the hard matching scale $\mu_{h}^{0}=m_{\phi}$ for both color sextet and antitriplet. Figure 3 shows the dependence of the hard function on $\mu_{h}$. It can be seen that the hard matching scale dependence decreases significantly from

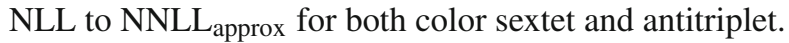

Fig. 2 The NLO total cross section for colored scalar production at the LHC with $\sqrt{s}=14 \mathrm{TeV}$. CTEQ6.1 [37] PDF sets are used. In the lower plots, the red solid lines represent the results in Ref. [6]
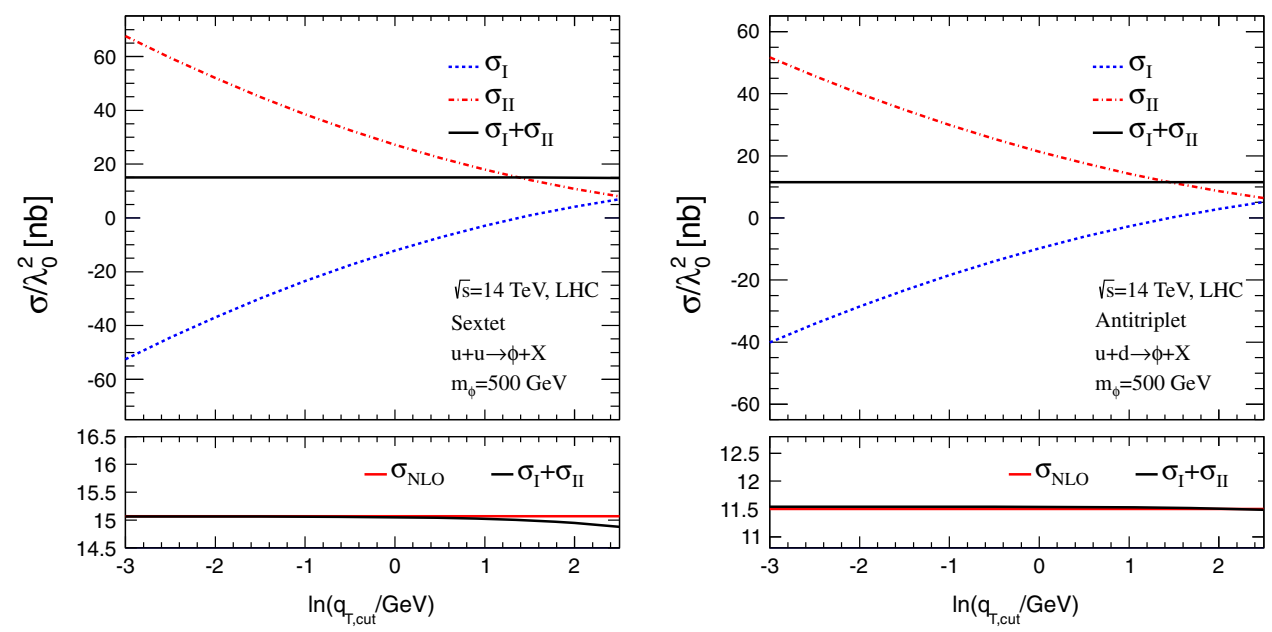
Fig. 3 Dependence of the hard function on hard matching scale $\mu_{h}$. For comparison, we show the dependence on $\mu_{h}$ for both NLL (dashed) and NNLL approx (solid) resummation
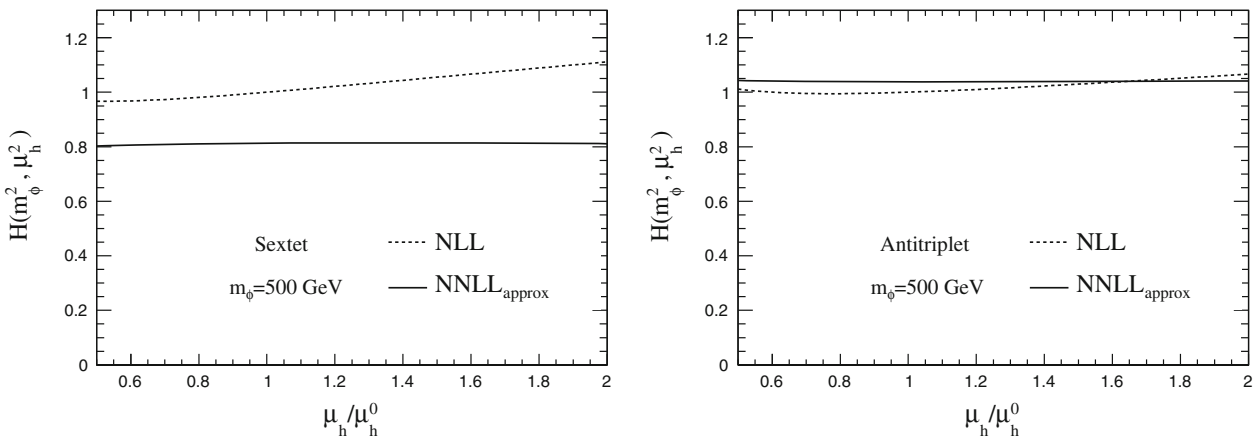

Fig. 4 Long-distance effects on the differential cross sections $\mathrm{d} \sigma / \mathrm{d} q_{\mathrm{T}}\left(\mathrm{NNLL}_{\text {approx }}\right)$. The left figure is for sextet and the right one is for antitriplet
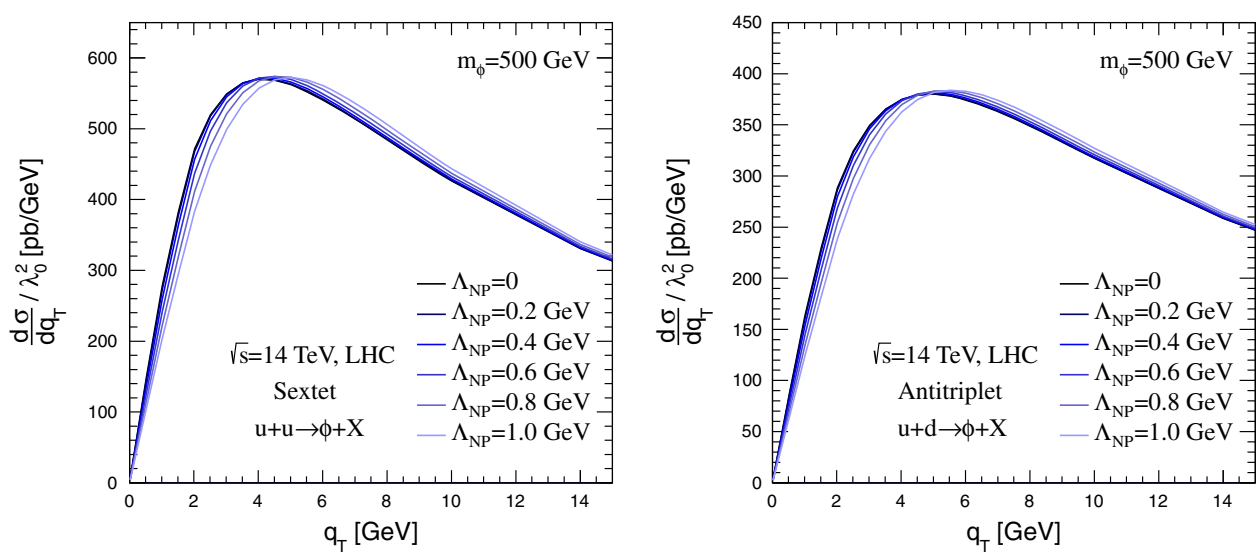

Fig. 5 Results of resummation for the transverse momentum distribution of single colored scalar production at $m_{\phi}=500 \mathrm{GeV}$ at the $\mathrm{LHC}$ with NLL (blue bands) and NNLL $_{\text {approx }}$ (red bands) accuracy. The thick lines represent the default scale choice

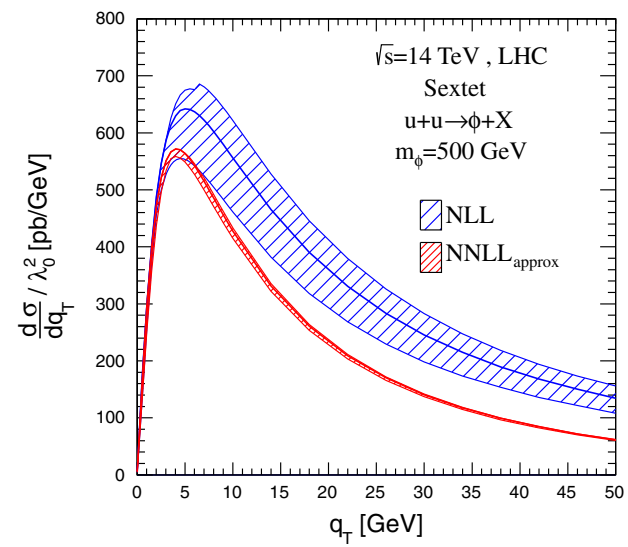

Our factorization formula is formally valid in the region $\Lambda_{\mathrm{QCD}} \ll q_{\mathrm{T}} \ll m_{\phi}$. When $q_{\mathrm{T}} \sim \Lambda_{\mathrm{QCD}}$, there are corrections in powers of $x_{\mathrm{T}} \Lambda_{\mathrm{QCD}}$, which comes form the operatorproduct expansion of the transverse PDFs [28]. These power corrections are of non-perturbative origin and one must model them, using some technique shown as follows [28]. The TMD PDFs are replaced by

$B_{q / N}\left(\xi, x_{\mathrm{T}}^{2}, \mu\right)=f_{\text {hadr }}\left(x_{\mathrm{T}} \Lambda_{\mathrm{NP}}\right) B_{q / N}^{\text {pert }}\left(\xi, x_{\mathrm{T}}^{2}, \mu\right)$,

where $\Lambda_{\mathrm{NP}}$ is a hadronic scale, and $f_{\text {hadr }}\left(x_{\mathrm{T}} \Lambda_{\mathrm{NP}}\right)$ is

$f_{\text {hadr }}\left(x_{\mathrm{T}} \Lambda_{\mathrm{NP}}\right)=\exp \left(-\Lambda_{\mathrm{NP}}^{2} x_{\mathrm{T}}^{2}\right)$.
Figure 4 shows the $\Lambda_{\mathrm{NP}}$ dependence of the results of transverse momentum resummation of single colored scalar production at the LHC with $\sqrt{s}=14 \mathrm{TeV}$. The non-perturbative form factor results in a small shift of the position of the peak of the $q_{\mathrm{T}}$ distribution. In addition, the $q_{\text {peak }}$ of color antitriplet is a little larger than the one of sextet. In the following calculation, we choose $\Lambda_{\mathrm{NP}}=600 \mathrm{MeV}$ [28] to simulate the non-perturbative effects for single colored scalar production.

In Fig. 5, we show the scale dependence of the differential cross section at the NLL and the NNLL ${ }_{\text {approx }}$, varying the factorization scale $\mu$ by a factor of 2 . It can be seen that the scale uncertainties reduce significantly from NLL to NNLL approx 
Fig. 6 Transverse momentum distribution for the single colored scalar production with mass of $500 \mathrm{GeV}$ and $1 \mathrm{TeV}$ at the LHC. The results in the traditional framework developed by CSS are shown with red dashed lines
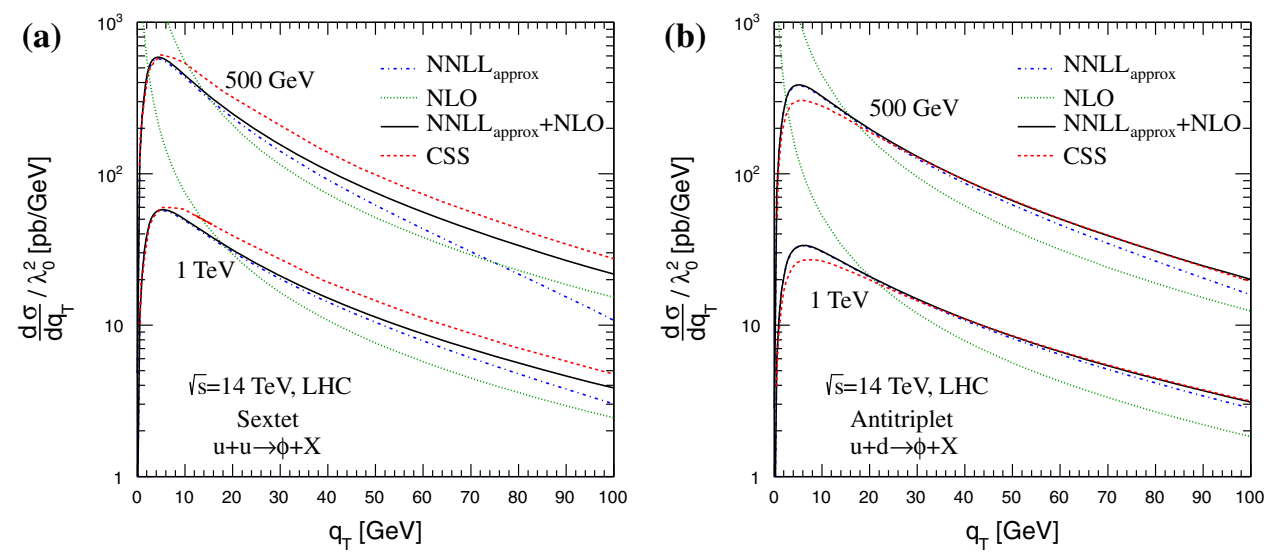

Fig. 7 PDF uncertainties for the production of colored scalar particles at the LHC. The bands correspond to $1 \sigma$ deviation variations of the PDF sets in MSTW2008 NNLO (red) and CT10 NNLO (green). In the lower row we show the deviations to each central value
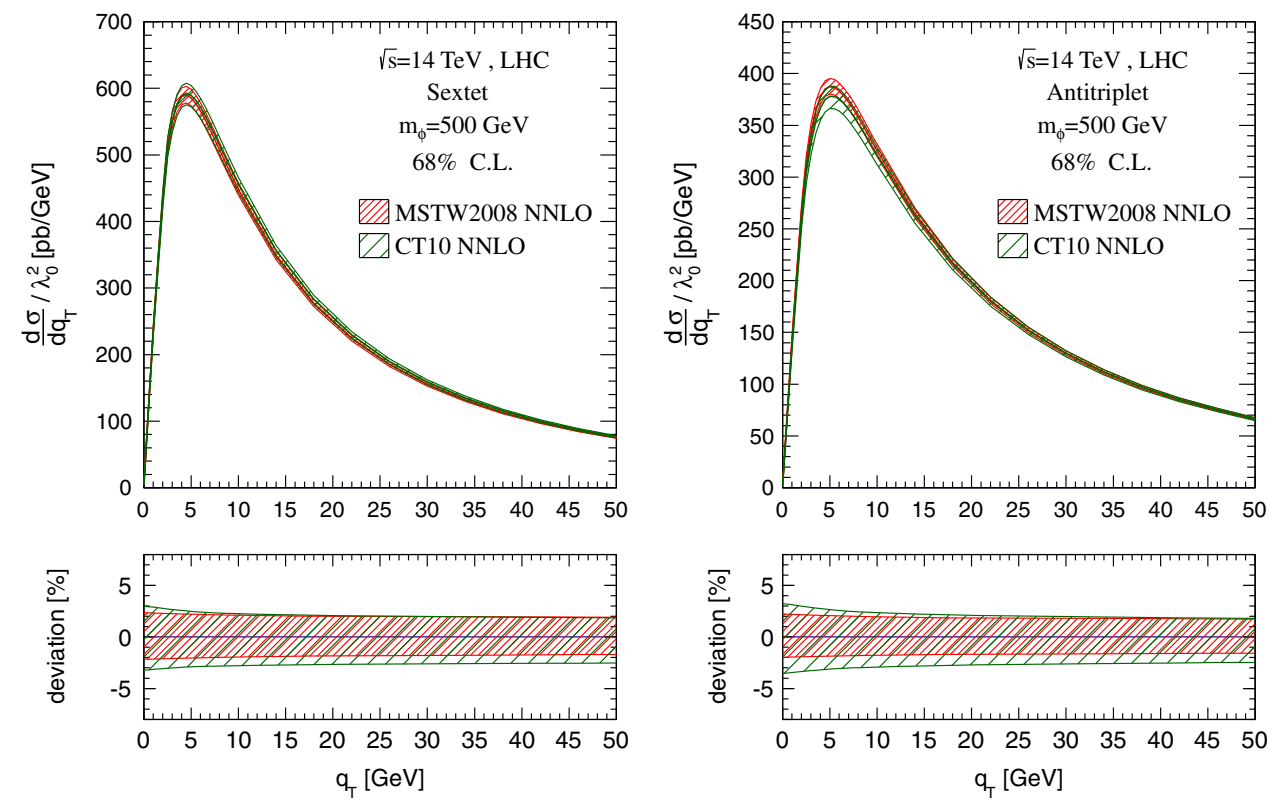

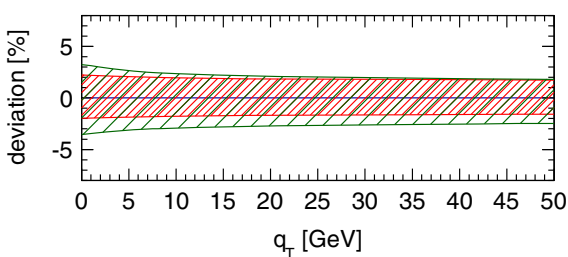

for both color sextet and antitriplet. In addition, comparing to NLL, the differential cross section at NNLL $\mathrm{Naprox}_{\text {in }}$ the peak region is suppressed for color sextet and enhanced for antitriplet. However, compared to the color antitriplet case, the NLL result has a larger deviation from the NNLLapprox for color sextet. It is because in NLL calculations the LO hard

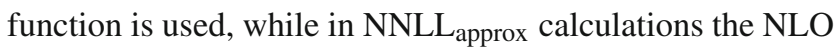
hard function is used, which gives a larger NLO correction to LO hard function (about $-20 \%$, as shown in Fig. 3) for color sextet. Thus, the theoretical prediction of NLL result for color sextet is inaccurate.

Figure 6 shows the transverse momentum distribution for both $m_{\phi}=500 \mathrm{GeV}$ and $m_{\phi}=1 \mathrm{TeV}$. Comparing with the work in Ref. [6], our results have some differences. First, the peak of the $q_{\mathrm{T}}$ distribution of NNLL approx $+\mathrm{NLO}$ is suppressed by about $3 \%$ for sextet and enhanced by about $25 \%$ for antitriplet. Second, our low $q_{\mathrm{T}}$ distributions peak around $4 \sim 6.5 \mathrm{GeV}$, while the peak region in Ref. [6] is around $5 \sim 8 \mathrm{GeV}$. These are due to the fact that our result of the resummation is presented at higher order than the one in Ref. [6].

In Fig. 7, we show the PDF uncertainties of the $q_{\mathrm{T}}$ distributions with MSTW2008NNLO [39] and CT10NNLO [40] PDF sets. For MSTW2008NNLO, the deviations are $\pm 2.5 \%$ for $q_{\mathrm{T}} \leq 5 \mathrm{GeV}$ and decrease to roughly $\pm 1 \%$ near $q_{\mathrm{T}}=50 \mathrm{GeV}$, while for CT10NNLO, the PDF uncertainties are a little larger. The central values of differential cross sections with the two different PDF sets are almost identical to each other, with deviation smaller than $\pm 2.5 \%$.

\section{Conclusion}

We have studied the factorization and transverse momentum resummation effects in the single production of the color sextet (antitriplet) scalars at the LHC with the SCET. The soft function is calculated in analytic regularization at the NLO and its validity is demonstrated. From the compari- 
son of the results for NLL and NNLL ${ }_{\text {approx }}$ resummation, we find the scale dependence is improved significantly in higher order. Comparing with the results in Ref. [6], the peak of the $q_{\mathrm{T}}$ distribution of NNLL $\mathrm{Naprox}+\mathrm{NLO}$ is suppressed by about $3 \%$ for sextet and enhanced by about $25 \%$ for antitriplet, respectively. In addition, our low $q_{\mathrm{T}}$ distributions peak around $4 \sim 6.5 \mathrm{GeV}$, while the peak region in Ref. [6] is around $5 \sim 8 \mathrm{GeV}$. Also, we discuss the long-distance corrections to the transverse momentum spectrum, and we show that they shift the peak positions about $0.2 \mathrm{GeV}$ with $\Lambda_{\mathrm{NP}}=600 \mathrm{MeV}$. Finally, we show that the PDF uncertainties are of order $\pm 2.5 \%$ in the peak region.

Acknowledgments We would like to thank Qing Hong Cao, Jian Wang and Ding Yu Shao for helpful discussions. This work was supported in part by the National Natural Science Foundation of China under Grants No. 11375013 and No. 11135003.

Open Access This article is distributed under the terms of the Creative Commons Attribution License which permits any use, distribution, and reproduction in any medium, provided the original author(s) and the source are credited.

Funded by $\mathrm{SCOAP}^{3}$ / License Version CC BY 4.0.

\section{References}

1. J.C. Pati, A. Salam, Phys. Rev. D 10, 275 (1974)

2. R.N. Mohapatra, R. Marshak, Phys. Rev. Lett. 44, 1316 (1980)

3. P. Fileviez, Perez. Phys. Lett. B 654, 189 (2007). arXiv:hep-ph/ 0702287

4. R. Barbier, C. Berat, M. Besancon, M. Chemtob, A. Deandrea et al., Phys. Rept. 420, 1 (2005). arXiv:hep-ph/0406039

5. R. Mohapatra, N. Okada, H.-B. Yu, Phys. Rev. D 77, 011701 (2008). arXiv:0709.486

6. T. Han, I. Lewis, T. McElmurry, JHEP 123, 1001 (2010). arXiv: 0909.2666

7. S. Chatrchyan et al., CMS Collaboration, (2013). arXiv:1302.4794

8. G. Aad et al., ATLAS Collaboration, Phys. Rev. D 86, 091103 (2012). arXiv:1209.6593

9. J.L. Hewett, T.G. Rizzo, Phys. Rep. 183, 193 (1989)

10. C.-H. Chen, Phys. Lett. B 680, 133 (2009). arXiv:0902.2620

11. C.-R. Chen, W. Klemm, V. Rentala, K. Wang, Phys. Rev. D 79, 054002 (2009). arXiv:0811.2105

12. T. Han, I. Lewis, Z. Liu, JHEP 085, 1012 (2010). arXiv: 1010.4309

13. S. Atag, O. Cakir, S. Sultansoy, Phys. Rev. D 59, 015008 (1999)

14. E. Arik, O. Cakir, S. Cetin, S. Sultansoy, JHEP 0209, 024 (2002). arXiv:hep-ph/0109011
15. O. Cakir, M. Sahin, Phys. Rev. D 72, 115011 (2005). arXiv:hep-ph/ 0508205

16. P. Richardson, D. Winn, Eur. Phys. J. C 72, 2012 (1862). arXiv: 1108.6154

17. I. Gogoladze, Y. Mimura, N. Okada, Q. Shafi, Phys. Lett. B 686, 233 (2010). arXiv: 1001.5260

18. E. L. Berger, Q.-H. Cao, C.-R. Chen, G. Shaughnessy, H. Zhang, Phys. Rev. Lett. 105, 181802 (2010). arXiv: 1005.2622

19. D. Karabacak, S. Nandi, S.K. Rai, Phys. Rev. D 85, 075011 (2012). arXiv: 1201.2917

20. Y.C. Zhan, Z.L. Liu, S.A. Li, C.S. Li, H.T. Li (2013). arXiv:1305. 5152

21. J.C. Collins, D.E. Soper, Nucl. Phys. B 193, 381 (1981)

22. J.C. Collins, D.E. Soper, Nucl. Phys. B 197, 446 (1982)

23. J.C. Collins, D.E. Soper, G.F. Sterman, Nucl. Phys. B 250, 199 (1985)

24. C.W. Bauer, S. Fleming, D. Pirjol, I.W. Stewart, Phys. Rev. D 63, 114020 (2001). arXiv:hep-ph/0011336

25. C.W. Bauer, D. Pirjol, I.W. Stewart, Phys. Rev. D 65, 054022 (2002). arXiv:hep-ph/0109045

26. M. Beneke, A. Chapovsky, M. Diehl, T. Feldmann, Nucl. Phys. B 643, 431 (2002). arXiv:hep-ph/0206152

27. T. Becher, M. Neubert, Eur. Phys. J. C 71, 1665 (2011). arXiv: 1007.4005

28. T. Becher, M. Neubert, D. Wilhelm, JHEP 1202, 124 (2012). arXiv: 1109.6027

29. H.X. Zhu, C.S. Li, H.T. Li, D.Y. Shao, L.L. Yang, Phys. Rev. Lett. 110, 082001 (2013). arXiv:1208.5774

30. H.T. Li, C.S. Li, D.Y. Shao, L.L. Yang, H.X. Zhu, Phys. Rev. D 88, 074004 (2013). arXiv: 1307.2464

31. J. Chay, C. Kim, Y.G. Kim, J.-P. Lee, Phys. Rev. D 71, 056001 (2005). arXiv:hep-ph/0412110

32. G. Korchemsky, A. Radyushkin, Phys. Lett. B 279, 359 (1992). arXiv:hep-ph/9203222

33. T. Becher, M. Neubert, Phys. Rev. D 79, 125004 (2009). arXiv: 0904.1021

34. T. Becher, M. Neubert, G. Xu, JHEP 0807, 030 (2008). arXiv:0710. 0680

35. S. Bosch, B. Lange, M. Neubert, G. Paz, Nucl. Phys. B 699, 335 (2004). arXiv:hep-ph/0402094

36. F. De Fazio, M. Neubert, JHEP 9906, 017 (1999). arXiv:hep-ph/ 9905351

37. D. Stump, J. Huston, J. Pumplin, W.-K. Tung, H. Lai et al., JHEP 0310, 046 (2003). arXiv:hep-ph/0303013

38. A. Martin, W. Stirling, R. Thorne, G. Watt, Eur. Phys. J. C 63, 189 (2009). arXiv:0901.0002

39. A. Martin, W. Stirling, R. Thorne, G. Watt, Eur. Phys. J. C 64, 653 (2009). arXiv:0905.3531

40. H.-L. Lai, M. Guzzi, J. Huston, Z. Li, P.M. Nadolsky et al., Phys. Rev. D 82, 074024 (2010). arXiv: 1007.2241 